\title{
Hemorragia espontánea de origen incierto
}

\author{
Ana Elvira-Laffond', David González-Calle', Miguel Hernández-Hidalgo', Ángel Víctor Hernández-Martos', María Sánchez-Ledesma² \\ 'Servicio de Cardiología. Complejo Asistencial Universitario de Salamanca. Salamanca. España \\ ${ }_{2}^{2}$ Servicio de Medicina Interna. Complejo Asistencial Universitario de Salamanca. Salamanca. España
}

Recibido:01/03/2020

Aceptado: 03/07/2020

En línea: 31/08/2020

Citar como: Elvira-Laffond A, González-Calle D, Hernández-Hidalgo M, Hernández-Martos AV, Sánchez-Ledesma M. Hemorragia espontánea de origen incierto. Rev Esp Casos Clin Med Intern (RECCMI). 2020 (Ago); 5(2): 74-77. doi: 10.32818/reccmi.a5n2a7.

Cite this as: Elvira-Laffond A, González-Calle D, Hernández-Hidalgo M, Hernández-Martos AV, Sánchez-Ledesma M. A spontaneous bleeding of uncertain origin. Rev Esp Casos Clin Med Intern (RECCMI). 2020 (Ago); 5(2): 74-77. doi: 10.32818/reccmi.a5n2a7.

Autor para correspondencia: Ana Elvira-Laffond. anaelaffond@gmail.com

Palabras clave
$\triangleright$ Hemofilia A
$\triangleright$ Factor VIII
$\triangleright$ Trastorno de la coagulación
$\triangleright$ Autoanticuerpos

\section{Keywords}

$\triangleright$ Hemophilia A

$\triangleright$ Factor VIII

$\triangleright$ Hemorrhagic disorders

$\triangleright$ Autoantibodies

\begin{abstract}
Resumen
La hemofilia A adquirida es un trastorno infrecuente de la coagulación debido a autoanticuerpos frente al factor VIII. Se debe sospechar en caso de sangrado espontáneo y prolongación del TTPA. Presentamos el caso de un varón de 85 años con hematoma retroperitoneal. En los análisis de laboratorio destacaba TTPA prolongado. Se determinó la actividad del factor VIII, que se encontraba significativamente disminuida. El paciente inició tratamiento, con excelente respuesta clínica. Este caso clínico es un ejemplo ilustrativo de esta enfermedad desconocida, que puede ayudar a su mejor comprensión y a un mejor manejo de estos pacientes.
\end{abstract}

Abstract
Acquired hemophilia A is a rare disease caused by autoantibodies targeting coagulation factor VIII. It
should be suspected in case of spontaneous bleeding and extended aPTT. We present an 85-year-old male
patient with retroperitoneal hematoma and prolonged aPTT. Factor VIII activity is determined, which is
significantly reduced. The patient begins treatment, with excellent clinical response. This is an illustrative
example of this unknown disease, which may provide its better understanding and management of these
patients.

\section{Puntos destacados}

$\triangleright$ Este caso ilustra la presentación clínica característica de la hemofilia A adquirida: hematoma espontáneo y prolongación del TTPA en un varón anciano, con comorbilidades.

$\triangleright$ Es un ejemplo excelente de un cuadro clínico infrecuente, que puede ayudar en su diagnóstico de forma precoz y en su tratamiento.

\section{Introducción}

La hemofilia A adquirida es un trastorno de la coagulación infrecuente con autoanticuerpos dirigidos frente al factor VIII. Suele manifestarse por la presencia de hemorragias espontáneas o sangrado prolongado tras intervenciones quirúrgicas, traumatismos o procedimientos invasivos, y ocurre en pacientes sin antecedentes familiares de trastornos de la coagulación. Su incidencia es variable, probablemente condicionada por la baja sospecha clínica de la enfermedad, lo que conlleva infradiagnóstico La mayoría de los casos descritos ocurren en pacientes añosos, con múl- tiples comorbilidades. La hemofilia adquirida es con mayor frecuencia de origen idiopático (40-50\%), aunque también puede deberse a la existencia de neoplasias, enfermedades autoinmunes, infecciones y algunos fármacos (Tabla 1$)^{1}$.

A diferencia de la hemofilia A hereditaria, las hemorragias suelen afectar a la piel, los músculos o los tejidos blandos, siendo infrecuente el hemartros. Con menor frecuencia puede existir sangrado genitourinario o retroperitoneal (< $10 \%)^{2}$. El riesgo de sangrado no se correlaciona con los niveles de factor VIII, y hay pacientes que pueden presentar sangrado significativo a pesar de tener niveles moderadamente reducidos del factor ${ }^{3}$.

Hay que sospechar hemofilia adquirida en pacientes que presentan hemorragias espontáneas o sangrado prolongado en la presencia de TTPA alargado y TP normal ${ }^{4}$. El tratamiento con heparina y anticoagulantes de acción directa puede interferir con el diagnóstico, siendo útil la determinación del tiempo de trombina y de anti-Xa para su diferenciación ${ }^{5}$. Una vez excluida la interferencia con anticoagulantes, deberá determinarse la actividad del factor VIII. El algoritmo diagnóstico se resume en la Figura 1. 


\begin{tabular}{|c|c|}
\hline $\begin{array}{l}\text { Situaciones/enfermedades asociadas } \\
\text { con hemofilia adquirida }\end{array}$ & Ejemplos más frecuentes \\
\hline \multicolumn{2}{|l|}{ Idiopática (52\%) } \\
\hline Enfermedades neoplásicas (12\%) & $\begin{array}{l}\text { - Tumores de órgano sólido: adenocarcinomas (próstata, pulmón) } \\
\text { - Neoplasias hematológicas: síndromes linfoproliferativos (leucemia linfocítica crónica, linfoma no } \\
\text { Hodgkin), gammapatías monoclonales }\end{array}$ \\
\hline \multicolumn{2}{|l|}{ Embarazo y posparto (8\%) } \\
\hline Enfermedades infecciosas (4\%) & Infección aguda por VHC y VHB \\
\hline Inducida por fármacos (3\%) & $\begin{array}{l}\text { - Antibióticos: penicilina, sulfamidas } \\
\text { - Antiepilépticos: fenitoína } \\
\text { - Inmunomoduladores: interferón, fludarabina } \\
\text { - Otros: clopidogrel, AINE }\end{array}$ \\
\hline Enfermedades dermatológicas (1\%) & $\begin{array}{l}\text { - Psoriasis } \\
\text { - Pénfigo }\end{array}$ \\
\hline Otras situaciones/enfermedades (10\%) & $\begin{array}{l}\text { - Transfusión de hemoderivados } \\
\text { - Otras enfermedades }\end{array}$ \\
\hline
\end{tabular}

Tabla 1. Situaciones y enfermedades asociadas con el desarrollo de hemofilia adquirida. Los porcentajes han sido redondeados al entero a partir de los datos procedentes del European Acquired Haemophilia Registry ${ }^{1}$

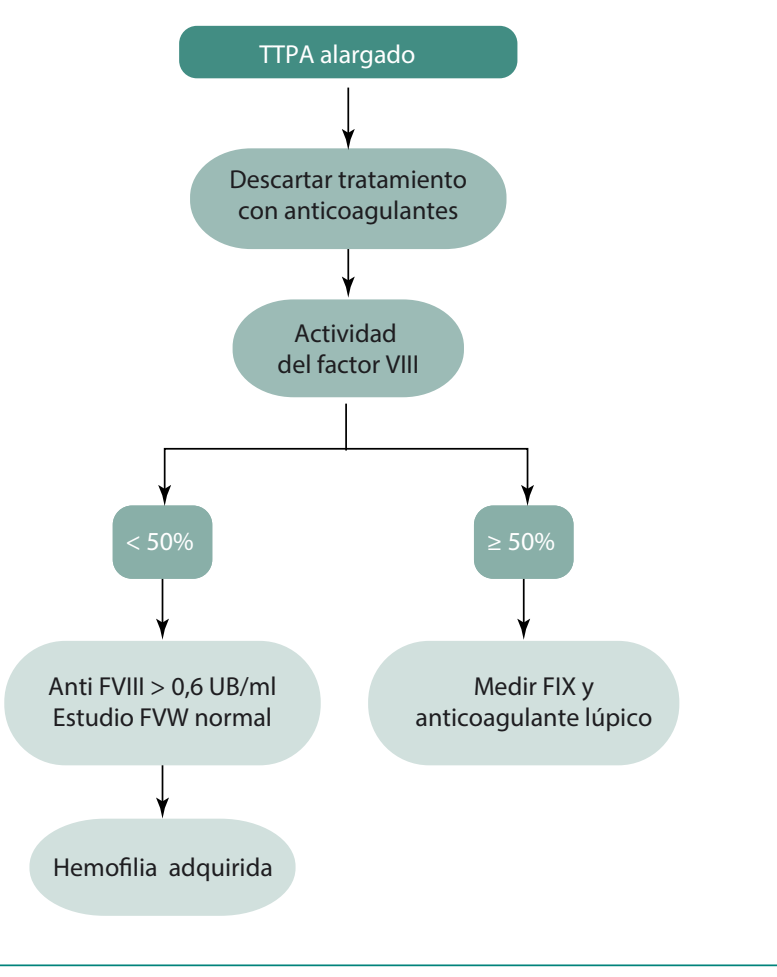

Figura 1. Algoritmo diagnóstico de la hemofilia adquirida

El tratamiento de la hemofilia adquirida comprende la terapia hemostática y la inmunosupresora. Todos aquellos pacientes con sangrado activo deberán recibir tratamiento hemostático ${ }^{6}$, siendo también necesario en pacientes con alto riesgo de sangrado (p. ej., intervención quirúrgica reciente). Las terapias hemostáticas disponibles se resumen en la Tabla 2.

En relación con el tratamiento inmunosupresor, debe emplearse en todos los pacientes con hemofilia adquirida, con el fin de eliminar el autoanticuerpo frente al factor VIII. El tratamiento de primera línea incluye el empleo de corticoides y ciclofosfamida, pudiéndose valorar el empleo de corticoides y rituximab como alternativa terapéutica? ${ }^{7}$.

\begin{tabular}{|c|c|c|}
\hline Tratamiento & Ventajas & Desventajas \\
\hline $\begin{array}{l}\text { Factor VIII } \\
\text { recombinante }\end{array}$ & $\begin{array}{l}\text { Posible monitorizar } \\
\text { su actividad de elección } \\
\text { en caso de hemorragia } \\
\text { con compromiso vital }\end{array}$ & $\begin{array}{l}\text { Menor eficacia } \\
\text { si autoanticuerpos } \\
\text { frente a rFVIII }\end{array}$ \\
\hline $\begin{array}{l}\text { - Agentes } \\
\text { "bypasseantes" } \\
\text { - Concentrado } \\
\text { de complejo } \\
\text { protrombínico } \\
\text { - Factor VII } \\
\text { recombinante }\end{array}$ & $\begin{array}{l}\text { Eficaces, no deben usarse } \\
\text { en caso de sangrado } \\
\text { con compromiso vital }\end{array}$ & $\begin{array}{l}\text { - Imposibilidad } \\
\text { - Rara monitorización } \\
\text { - } \text { Uiesgo de trombosis en caso } \\
\text { de títulos altos } \\
\text { de autoanticuerpos } \\
\text { frente a rFVIII }\end{array}$ \\
\hline
\end{tabular}

Tabla 2. Tratamiento hemostático de la hemofilia adquirida

\section{Caso clínico}

\section{Antecedentes}

Varón de 85 años que acudía al Servicio de Urgencias por rectorragia e impotencia funcional en miembro inferior izquierdo. El paciente refería que había presentado emisión de sangre roja fresca a través del ano, de forma espontánea. No presentaba clínica de prurito anal, tenesmo o alteraciones en el hábito intestinal o en las características de las heces. No había tenido clínica de sangrado a otros niveles. Por otro lado, refería pérdida de fuerza progresiva en el miembro inferior izquierdo, indolora y sin antecedente traumático previo.

- Antecedentes personales. Hipertensión arterial esencial y diabetes mellitus tipo 2, con nefropatía de origen mixto (nefroangioesclerosis y diabética) en estadio KDIGO IIIb. Enfermedad pulmonar obstructiva estadio GOLD D. Estudiado por anemia ferropénica con necesidades transfusionales frecuen- 
tes, con gastroscopia y colonoscopia normales y una única determinación de sangre oculta en heces positiva, con posteriores muestras negativas.

- Antecedentes familiares. Padre fallecido a los 72 años de infarto de miocardio. Madre fallecida de cáncer de ovario a los 81 años. Una hermana de 81 años, sana.

- Tratamiento habitual. Insulina glargina 12 UI, fluvastatina 40 mg, furosemida 40 mg, aclidinio/formoterol fumarato dihidrato 340/12 $\mu \mathrm{g}$.

\section{Exploración física}

- Tensión arterial: 160/65 mmHg. Frecuencia cardíaca: 98 lpm. SpO 95\%. Frecuencia respiratoria: $16 \mathrm{rpm}$.

- Buen estado general. Consciente y orientado. Palidez cutáneo-mucosa. Eupneico en reposo.

- Auscultación cardíaca. Tonos cardíacos rítmicos, sin soplos ni extratonos.

- Auscultación respiratoria. Murmullo vesicular conservado, con crepitantes de despegamiento bibasales.

- Abdomen muy globuloso, doloroso a la palpación en flanco izquierdo, sin masas ni visceromegalias. Ruidos intestinales normales.

- Exploración neurológica. Isocoria y normorreactividad pupilar. Pares craneales normales. Hipotrofia e hipotonía leve en miembros inferiores, sin asimetrías. Debilidad para la flexión de la cadera izquierda (4/5) y para la rotación (4+/5), sin alteraciones en el balance muscular del resto de extremidades. Sin alteraciones en la sensibilidad. Reflejos osteotendinosos presentes y simétricos. Reflejo plantar flexor. Sin alteraciones en la coordinación. Marcha con dificultad para la fase de despegue del miembro inferior izquierdo.

- Tacto rectal. Sin heces en la ampolla rectal. Tono de esfínter normal. Plexos hemorroidales no dilatados. Dedil con restos de heces de aspecto normal, sin sangre.

\section{Pruebas complementarias}

- Hemograma. Hemoglobina 6,5 g/dl, MCV 76,2 fl, MCH 24,5pg, MCHC 32,2g/ dl, RDW 21,8\%. Leucocitos 17,56 103/ml, neutrófilos 14,23 103/ml. Plaquetas $39610^{3} / \mathrm{ml}$.

- Bioquímica. Creatinina 3,2 mg/dl (estimación de FG por CKD-EPI 14 ml/ min). lonograma normal. Perfil hepático normal.

- Coagulación. TP 13 s, actividad de protrombina 81\%, INR 1,14, TTPA 68,6 segundos.

- Tomografía computarizada (TC) abdominal (sin contraste por enfermedad renal crónica agudizada). Aumento del compartimento ilio-psoas izquierdo, con densidad ligeramente heterogénea que sugiere hematoma retroperitoneal evolucionado (Figura 2).

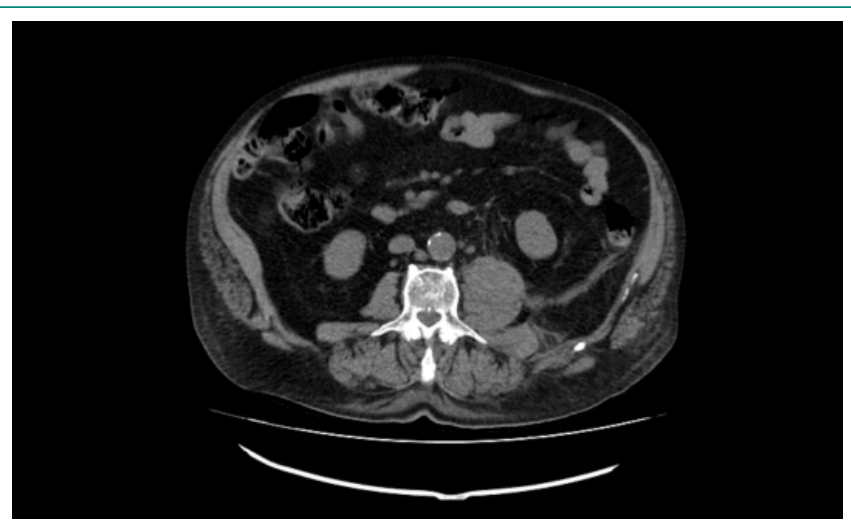

Figura 2. TC abdominal sin contraste que demuestra aumento del compartimento psoas-ilíaco izquierdo, de aspecto heterogéneo, sugestivo de hematoma retroperitoneal evolucionado

\section{Evolución}

El paciente ingresó hemodinámicamente estable en la planta de hospitalización de Medicina Interna. Se realizó colonoscopia de forma programada, que mostró como único hallazgo la presencia de hemorroides internas sin signos de complicación ni sangrado activo.

Ante la ausencia de factores que justificasen el sangrado, y el hallazgo de un TTPA prolongado en varias analíticas (actuales y previas) sin el consumo concomitante de fármacos anticoagulantes, se solicitó estudio de factores de la coagulación. En dicho estudio se objetivaron niveles de factor VIII: C del 1,17\%, con título de inhibidor de 6,72 UB/ml y anticoagulante lúpico negativo. Estos hallazgos confirmaron el diagnóstico de hemofilia adquirida.

Se inició tratamiento con factor VII recombinante, ciclofosfamida y corticoides, con excelente respuesta clínica. El paciente no presentó nuevos episodios de sangrado y, tras 1 semana con tratamiento, presentó niveles de factor VIII del 30\%, sin detectarse inhibidor. Se solicitaron TC cervical, estudio de autoinmunidad, marcadores tumorales y proteinograma para descartar un origen neoplásico o autoinmune de la enfermedad, que resultaron negativos.

El paciente alcanzó respuesta completa 2 meses después de haber comenzado el tratamiento, sin nuevos episodios de sangrado y con niveles de factor VIII superiores al 50\%.

\section{Diagnóstico}

Hemofilia A adquirida idiopática.

\section{Discusión y conclusión}

Se presenta un caso ilustrativo de hemofilia A adquirida, con las características típicas de esta enfermedad (paciente añoso, con comorbilidades, origen idiopático). La hemofilia adquirida es un trastorno de la coagulación infrecuente, aunque su baja prevalencia se pueda atribuir, en parte, a infradiagnóstico. Este paciente había tenido un seguimiento prolongado por anemia ferropénica, con necesidad de transfusión en varias ocasiones y sin haberse llegado a identificar la causa del sangrado. Sin embargo, presentaba analíticas de meses previos al episodio descrito en las que se objetivaba prolongación del TTPA.

Resulta fundamental difundir el conocimiento de esta enfermedad, con el fin de mejorar su diagnóstico y, con ello, ofrecer un tratamiento eficaz a estos pacientes.

\section{Bibliografía}

1. Knoebl P, Marco P, Baudo F, et al. Demographic and clinical data in acquired hemophilia A: results from the European Acquired Haemophilia Registry (EACH2). J Thromb Haemost. 2012; 10: 622-631.

2. Collins PW, Hirsch S, Baglin TP, et al. Acquired haemophilia A in the United Kingdom: a 2-year national surveillance study by the United Kingdom Haemophilia Centre Doctors' Organisation. Blood. 2008; 90: 18701877.

3. Delgado J, Jiménez-Yuste V, Hernández-Navarro F, Villar A. Acquired haemopholia: review and meta-analysis focused on tjerapy and prognostic factors. Br J Haematol. 2003; 121: 21-35 
4. Tiede A, Werwitzke S, Scharf RE. Laboratory diagnosis of acquired haemophilia A: limitations, consequences and challenges. Semin Throm Hemost. 2014; 40: 803-811.

5. Collins $P$, Baudo F, Huth-Kuhne A, et al. Consensus recommendations for the diagnosis and treatment of acquired hemophilia A. BMC Res Notes. 2010; 3: 161
6. Huth-Kuhne A, Baudo F, Collins P, et al. International recommendations on the diagnosis and treatment of patients with acquired haemophilia $A$. Haematologica. 2009; 94: 566-575.

7. Kessler CM, Knöbl P. Acquired haemophilia: an overview for clinical practice. Eur J Haematol. 2015 Dec; 95(Suppl 81): 36-44. 